\title{
Experimentelle Untersuchungen zur Frage des Verlaufes der oculo-pupillären Fasern in den hinteren Wurzeln.
}

\author{
(Vorläufige Mitteilung.) \\ Yon \\ E. Pollak und E. Sternschein. \\ (Aus dem neurologischen Institut der. Universität in Wien \\ [Vorstand: Prof. Marburg].) \\ (Eingegangen am 10. Oktober 1921.)
}

Durch die Untersuchungen W. Lehmanns ist die Frage nach dem Verlaufe der efferenten Fasern in den Hinterwurzeln wiederum aufgerollt worden. Bisnun ist eine sichere Entscheidung hierin noch nicht möglich gewesen, zumal hierher auch die verschiedenen Befunde über Tonus-Fasern gehören, welche durch die hinteren Wurzeln zum Erfolgsorgan ziehen sollen (E. Frank u. a.). Die in jüngster Zeit propagierte Beteiligung des Sympathicus resp. des autonomen Systems am $\mathrm{Zu}$ standekommen des Tonus (vgl. vor allem de Boer) führte uns aber zu den im folgenden kurz berichteten experimentelien Versuchen. Für uns lagen nämlich die Fragen vor, ob erstens die oculo-pupillären Fasern das Rückenmark durch die hinteren Wurzeln verlassen resp. ob den in den Spinalganglien eingeschalteten sympathischen Fasern ein Einfluß auf die Erhaltung eines bestimmten Dilatator-Tonus zukommt. $\mathrm{Zu}$ diesem Zwecke wurden vorläufig an zwei Kaninchen die hinteren Wurzeln in der Höhe von $\mathrm{C}_{5}-\mathrm{D}_{3}$ einseitig reseziert. Es zeigte sich, daß weder Folgeerscheinungen an der Pupille nachweisbar waren kurz nach der Operation, noch in den späteren Tagen. Die beiden Pupillen waren stets gleich weit geblieben.

Diese Versuche sollen beweisen, da $\beta$ die hinteren Wurzeln keinen Einfluß auf den Dilatator-Tonus der Pupille ausüben. Doch sollen erst weitere Untersuchungen an größerem Material die absolute Beweiskraft dieser Versuche erhärten, da immerhin eine individuelle Variationsbreite zugestanden werden muß, wenngleich bei dem Umfang des vorgenommenen Eingriffes dieser Einwand leicht zurückzuweisen wäre. 